\title{
Stability analysis of discrete-time multi-patch Beddington-DeAngelis type predator-prey model with time-varying delay
}

\author{
Jiqiang Feng ${ }^{1 *}$ and Zhiqiang Zhao ${ }^{2}$
}

"Correspondence:

fengjqszu@163.com

'Shenzhen Key Laboratory of

Advanced Machine Learning and

Application, College of Mathematics

and Statistics, Shenzhen University,

Shenzhen, China

Full list of author information is

available at the end of the article

\section{Springer}

\begin{abstract}
This paper is concerned with the stability of a discrete-time multi-patch Beddington-DeAngelis type predator-prey model with time-varying delay, where the dispersal of both predators and prey is considered. A nonstandard finite difference scheme is used to discretize this model. Then, combining the Lyapunov-Krasovskii method with the graph-theoretical technique, a stability criterion is derived, which is closely related to the dispersal topology. And an example with numerical simulation is given to demonstrate the effectiveness of the obtained results.
\end{abstract}

Keywords: Beddington-DeAngelis type predator-prey model; Time-varying delay; Nonstandard finite difference scheme; Multiple patch with multiple dispersal; Lyapunov-Krasovskii method

\section{Introduction}

A famous predator-prey system with Beddington-DeAngelis type response in the following form

$$
\begin{aligned}
& \frac{\mathrm{d} x(t)}{\mathrm{d} t}=x(t)\left(r-b x(t)-\frac{p y(t)}{1+u x(t)+v y(t)}\right), \\
& \frac{\mathrm{d} y(t)}{\mathrm{d} t}=y(t)\left(-\gamma-\delta y(t)+\frac{q x(t)}{1+u x(t)+v y(t)}\right),
\end{aligned}
$$

has been widely studied recently (see, e.g., [1-5]). In system (1), $x(t)$ and $y(t)$ represent the densities of prey and predators at time $t$, respectively, For biological significance of parameters $r, \gamma, b, \delta, p, q, u, v$, we refer the reader to $[6,7]$.

In practice, due to competition or foraging, species dispersal among multiple patches (groups) is an inevitable phenomenon. Most existing results that are concerned with predator-prey models only considered the prey disperse among $n(n>2)$ patches (see, e.g., $[8,9])$. In reality, not only prey but predators can disperse among $n$ patches. So considering the multiple dispersal situation is more practical. In $[10,11]$, continuous-time and discrete-time multi-patch predator-prey models with the dispersal of both predators and prey were considered, respectively. However, since time delay frequently occurs in almost every situation, it is essential to take time delay into account. And until now, few results

(c) The Author(s) 2019. This article is distributed under the terms of the Creative Commons Attribution 4.0 International License (http://creativecommons.org/licenses/by/4.0/), which permits unrestricted use, distribution, and reproduction in any medium, provided you give appropriate credit to the original author(s) and the source, provide a link to the Creative Commons license, and indicate if changes were made. 
concern multi-patch predator-prey model with time delay, especially the situation that both predators and prey disperse among multiple patch. And to our best knowledge, the method in $[10,11]$ cannot be used directly to cope with time delay.

Based on the above discussion, it is greatly meaningful to study the following Beddington-DeAngelis type predator-prey model with time-varying delay and multiple dispersal among $l$ patches:

$$
\left\{\begin{aligned}
\frac{\mathrm{d} x_{i}(t)}{\mathrm{d} t}= & x_{i}(t)\left(r_{i}-b_{i} x_{i}(t)-e_{i} y_{i}\left(t-\tilde{\tau}_{1}(t)\right)-\frac{p_{i} y_{i}(t)}{1+u_{i} x_{i}(t)+v_{i} y_{i}(t)}\right) \\
& +\sum_{j=1}^{l} a_{i j}\left(x_{j}(t)-\alpha_{i j} x_{i}(t)\right), \\
\frac{\mathrm{d} y_{i}(t)}{\mathrm{d} t}= & y_{i}(t)\left(-\gamma_{i}-\delta_{i} y_{i}(t)+\varepsilon_{i} x_{i}\left(t-\tilde{\tau}_{2}(t)\right)+\frac{q_{i} x_{i}(t)}{1+u_{i} x_{i}(t)+v_{i} y_{i}(t)}\right) \\
& +\sum_{j=1}^{l} b_{i j}\left(y_{j}(t)-\beta_{i j} y_{i}(t)\right), \quad t \geq 0, i \in \mathbb{L},
\end{aligned}\right.
$$

where $\mathbb{L}=\{1,2, \ldots, l\}, r_{i}, b_{i}, e_{i}, \ldots$ denote the corresponding parameters in patch $i$ and are all nonnegative constants, $\tilde{\tau}_{1}, \tilde{\tau}_{2}$ denote the time delays, $a_{i j}\left(x_{j}(t)-\alpha_{i j} x_{i}(t)\right)$ and $b_{i j}\left(y_{j}(t)-\right.$ $\left.\beta_{i j} y_{i}(t)\right)$ stand for the dispersal of prey and predators from patch $j$ to patch $i$ respectively. Constants $a_{i j}, b_{i j}$ are the dispersal rate, and the meaning of $\alpha_{i j}, \beta_{i j}$ can be seen in [12]. It is worth noting that few results have been reported on the stability of system (2).

Moreover, it is important and interesting to investigate discrete-time predator-prey model, especially when the size of population is rarely small or the population has no overlapping generation. In this paper, we construct a nonstandard finite difference scheme and apply it to system (2). Then, by simple calculation, one can have the explicit expression as follows:

$$
\left\{\begin{array}{l}
x_{i}(n+1)=\frac{x_{i}(n)+h\left(r_{i} x_{i}(n)+\sum_{j=1}^{l} a_{i j} x_{j}(n)\right)}{1+h\left(b_{i} x_{i}(n)+e_{i} y_{i}\left(n-\tau_{1}(n)\right)+\frac{p_{i} y_{i}(n)}{1+u_{i} x_{i}(n)+v_{y} y_{i}(n)}+\sum_{j=1}^{l} a_{i j} \alpha_{i j}\right)}, \\
y_{i}(n+1)=\frac{y_{i}(n)+h\left(\varepsilon_{i} y_{i}(n) x_{i}\left(n-\tau_{2}(n)\right)+\frac{q_{i} x_{i}(n) y_{i}(n)}{1+u_{i} x_{i}(n)+v_{i} y_{i}(n)}+\sum_{j=1}^{l} b_{i j} y_{j}(n)\right)}{1+h\left(\gamma_{i}+\delta_{i} y_{i}(n)+\sum_{j=1}^{l} b_{i j} \beta_{i j}\right)},
\end{array}\right.
$$

where $i \in \mathbb{L}, h>0$ is the time step size, and $\tau_{1}(n)=\left[\frac{\tilde{\tau}_{1}(n h)}{h}\right], \tau_{2}(n)=\left[\frac{\tilde{\tau}_{2}(n h)}{h}\right]$, where $[a]$ represents the integer part of $a \in \mathbb{R}_{+}^{1}, x_{i}(n)$ and $y_{i}(n)$ are the numerical approximations of $x_{i}$ and $y_{i}$ at $t_{n}=n h$, respectively. Clearly, the solutions of system (3) are positive unconditionally if the initial conditions are positive.

In this paper, a systematic method is provided to construct a global LyapunovKrasovskii function for system (3), that is the combination of Lyapunov-Krasovskii function of each patch and the graph-theoretical technique on multiple digraphs. Then a criterion is derived to ensure the stability of system (3), which is closely related to the topological structure of the dispersal networks and the bound of the time-varying delay. Finally, an example showing the effectiveness of the provided results is given.

\section{Main results}

Let $\mathbb{N}=\{0,1,2, \ldots\}, \mathbb{R}_{+}=[0,+\infty)$, and $\mathbb{L}=\{1,2, \ldots, l\}$. Write $\mathbb{R}^{m}$ for an $m$-dimensional Euclidean space and denote by $\mathbb{R}_{+}^{m}=\left\{\left(y_{1}, y_{2}, \ldots, y_{m}\right)^{\mathrm{T}} \in \mathbb{R}^{m}: y_{i}>0, i=1,2, \ldots, m\right\}$. Define $\mathbb{S}_{\delta}^{m}\left(x^{*}\right)=\left\{x \in \mathbb{R}^{m}:\left|x-x^{*}\right|<\delta\right\}$. The nonnegative function $\tau(n)$ denotes the time delay, satisfying $\tau_{m} \leq \tau(n) \leq \tau_{M}, n \in \mathbb{N}$, where $\tau_{m}$ and $\tau_{M}$ are positive integers.

A digraph $\mathcal{G}$ can be represented by $\mathcal{G}=(\mathcal{U}, \mathcal{V})$, where $\mathcal{U}=\mathbb{L}$ is a vertices set and $\mathcal{V}$ is an arcs set. Arc $(i, j) \in \mathcal{V}$ stands for an arc leading from vertex $i$ to vertex $j$ and $(i, i) \notin \mathcal{V}$ for 
all $i \in \mathcal{U}$. A digraph $\mathcal{G}$ is weighted if each arc $(j, i)$ has a positive weight $a_{i j}$ and $a_{i j}>0$ if and only if there exists an $\operatorname{arc}(i, j)$ in $\mathcal{G}$. Denote by $A=\left(a_{i j}\right)_{l \times l}$ the weight matrix of $\mathcal{G}$. We mean $(\mathcal{G}, A)$ as a digraph $\mathcal{G}$ with weight matrix $A$. Define the Laplacian matrix of $\mathcal{G}$ to be $L(\mathcal{G})=\left(\varphi_{i j}\right)_{l \times l}$, where

$$
\varphi_{i j}= \begin{cases}\sum_{r \neq i} a_{i r}, & i=j, \\ -a_{i j}, & i \neq j .\end{cases}
$$

For other details on graph theory, the reader can be referred to [13].

Without loss of generality, assume system (3) has a unique positive equilibrium

$$
X^{*}=\left(\left(X_{1}^{*}\right)^{\mathrm{T}},\left(X_{2}^{*}\right)^{\mathrm{T}}, \ldots,\left(X_{l}^{*}\right)^{\mathrm{T}}\right)^{\mathrm{T}},
$$

where $X_{i}^{*}=\left(x_{i}^{*}, y_{i}^{*}\right)^{\mathrm{T}}$. Furthermore, we introduce $X_{i}^{(n)}=\left(x_{i}(n), y_{i}(n)\right)^{\mathrm{T}}$ and $X_{n}=\left(\left(X_{1}^{(n)}\right)^{\mathrm{T}}\right.$, $\left.\left(X_{2}^{(n)}\right)^{\mathrm{T}}, \ldots,\left(X_{l}^{(n)}\right)^{\mathrm{T}}\right)^{\mathrm{T}}$. Let $\tilde{a}_{i j}=a_{i j} q_{i}\left(1+v_{i} y^{*}\right) x_{j}^{*}, \tilde{b}_{i j}=b_{i j} p_{i}\left(1+u_{i} x^{*}\right) y_{j}^{*}, \tilde{A}=\left(\tilde{a}_{i j}\right)_{l \times l}$, and $\tilde{B}=$ $\left(\tilde{b}_{i j}\right)_{l \times l}$. Moreover, denote by $c_{1}^{(i)}$ and $c_{2}^{(i)}$ the cofactor of the $i$ th diagonal element of the Laplacian matrix of $\left(\mathcal{G}_{1}, \tilde{A}\right)$ and $\left(\mathcal{G}_{2}, \tilde{B}\right)$, respectively. In this paper, we establish system (3) on diagraphs $\left(\mathcal{G}_{1}, \tilde{A}\right)$ and $\left(\mathcal{G}_{2}, \tilde{B}\right)$. In $\left(\mathcal{G}_{1}, \tilde{A}\right)$, the vertices represent prey and arcs denote the dispersal of prey. In $\left(\mathcal{G}_{2}, \tilde{B}\right)$, the vertices represent predators and arcs denote the dispersal of predators. In order to better illustrate our model, we present an illustrative diagram for the dispersal of both prey and predators as shown in Fig. 1.

From Fig. 1, we can see that there are two dispersal networks for our model, which, respectively, illustrate the dispersal of prey and predators. While in many previous results, see $[8,9]$ for example, the authors only considered the dispersal of prey. In this case, the illustrative diagram becomes the form of Fig. 2. While in reality, the dispersal of prey and predators among patches should be consistent. Hence, considering the dispersal of prey and predators simultaneously is more realistic. Another illustrative example is the epidemic model in a patchy environment [14], in which both susceptible individuals and infectious individuals disperse among patches.

Then a stability criterion for system (3) is given as follows.

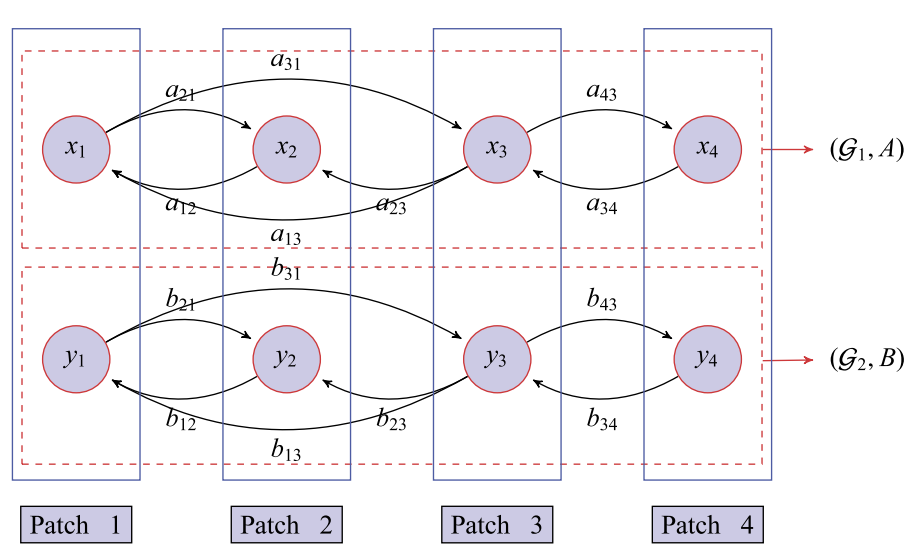

Figure 1 A structure for system (3) on four patches with two weighted digraphs: digraph $(\mathcal{G}, \tilde{A})$ represents the dispersal of prey and digraph $(\mathcal{G}, \tilde{B})$ represents the dispersal of predators 


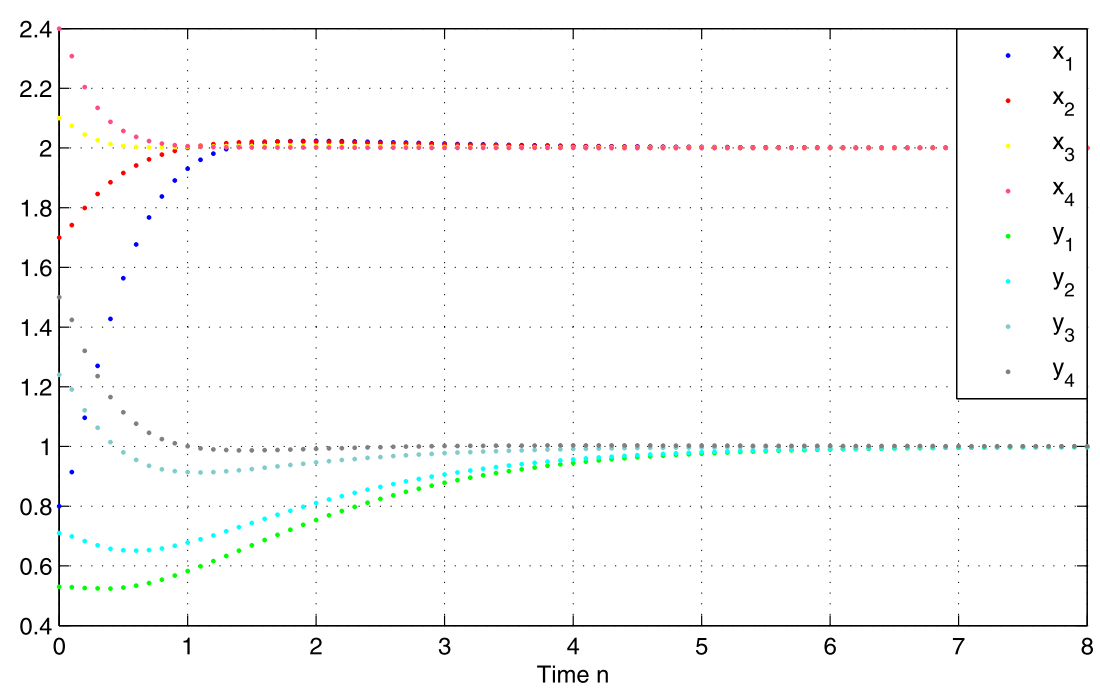

Figure 2 A structure for system (3) on four patches with the dispersal of prey denoted by $\operatorname{digraph}(\mathcal{G}, \tilde{A})$

Theorem 1 Suppose that digraphs $\left(\mathcal{G}_{1}, \tilde{A}\right)$ and $\left(\mathcal{G}_{2}, \tilde{B}\right)$ are strongly connected and there exists $\theta>0$ such that $c_{1}^{(i)}=\theta c_{2}^{(i)}$ for any $i \in \mathbb{L}$. Suppose that there exist positive constants $\sigma_{1}^{(i)}$ and $\sigma_{2}^{(i)}$ satisfying the following inequality:

$$
\frac{\varepsilon_{i}^{2} h}{2 \sigma_{1}^{(i)}}<\theta<\frac{2 \sigma_{2}^{(i)}}{e_{i}^{2} h}
$$

and

$$
\begin{aligned}
& \lambda_{1}^{(i)} h\left(\frac{p_{i} u_{i} y_{i}^{*}}{1+u_{i} x_{i}^{*}+v_{i} y_{i}^{*}}+\frac{\lambda_{1}^{(i)}}{2}-b_{i}\right)+\sigma_{1}^{(i)}\left(1+\tau_{M}^{(2)}-\tau_{m}^{(2)}\right)<0, \\
& \lambda_{2}^{(i)} h\left(\frac{\lambda_{2}^{(i)}}{2}-\delta_{i}\right)+\sigma_{2}^{(i)}\left(1+\tau_{M}^{(1)}-\tau_{m}^{(1)}\right)<0, \quad i \in \mathbb{L}
\end{aligned}
$$

where $\lambda_{1}^{(i)}=q_{i}\left(1+v_{i} y^{*}\right)$ and $\lambda_{2}^{(i)}=\theta p_{i}\left(1+u_{i} x^{*}\right)$. Then, for any $\varepsilon$ and $M$ satisfying $0<\varepsilon<M$, there exist $\tilde{h}(\varepsilon, M)>0$ and $\mathbb{S}_{M}^{2 l}\left(X^{*}\right) \in \mathbb{R}_{+}^{2 l}$ such that, for any $h \in(0, \tilde{h})$,

$$
\limsup _{n \rightarrow \infty}\left|X_{n}-X^{*}\right|<\varepsilon, \quad X_{0} \in \mathbb{S}_{M}^{2 l}\left(X^{*}\right)
$$

Proof Define a Lyapunov function for system (3) as follows:

$$
V\left(X_{n}\right)=\sum_{i=1}^{l}\left(c_{1}^{(i)} \sum_{r=1}^{3} V_{1, r}^{(i)}\left(x_{i}(n)\right)+c_{2}^{(i)} \sum_{r=1}^{3} V_{2, r}^{(i)}\left(y_{i}(n)\right)\right),
$$

where

$$
\begin{aligned}
& V_{1,1}^{(i)}\left(x_{i}(n)\right)=\lambda_{1}^{(i)}\left(x_{i}(n)-x_{i}^{*} \ln x_{i}(n)\right), \\
& V_{2,1}^{(i)}\left(y_{i}(n)\right)=\lambda_{2}^{(i)}\left(y_{i}(n)-y_{i}^{*} \ln y_{i}(n)\right),
\end{aligned}
$$


Fens and Zhao Advances in Difference Equations

(2019) 2019:444

Page 5 of 12

$$
\begin{aligned}
& V_{1,2}^{(i)}\left(x_{i}(n)\right)=\sigma_{1}^{(i)} \sum_{r=n-\tau_{2}(n)}^{n-1}\left(x_{i}(r)-x_{i}^{*}\right)^{2}, \\
& V_{2,2}^{(i)}\left(y_{i}(n)\right)=\sigma_{2}^{(i)} \sum_{r=n-\tau_{1}(n)}^{n-1}\left(y_{i}(r)-y_{i}^{*}\right)^{2}, \\
& V_{1,3}^{(i)}\left(x_{i}(n)\right)=\sigma_{1}^{(i)} \sum_{q=n-\tau_{M}^{(2)}+1}^{n-\tau_{m}^{(2)}} \sum_{r=q}^{n-1}\left(x_{i}(r)-x_{i}^{*}\right)^{2}, \\
& V_{2,3}^{(i)}\left(y_{i}(n)\right)=\sigma_{2}^{(i)} \sum_{q=n-\tau_{M}^{(1)}+1}^{n-\tau_{m}^{(1)}} \sum_{r=q}^{n-1}\left(y_{i}(r)-y_{i}^{*}\right)^{2} .
\end{aligned}
$$

It is easy to show that $V\left(X_{n}\right) \in C^{1}\left(\mathbb{R}^{m l} ; \mathbb{R}_{+}\right)$and $V\left(X^{*}\right)=0$.

Firstly, calculating $\Delta V_{1,1}^{(i)}\left(x_{i}(n)\right)$ along (3), one can arrive at

$$
\begin{aligned}
& \Delta V_{1,1}^{(i)}\left(x_{i}(n)\right) \\
& =V_{1,1}^{(i)}\left(x_{i}(n+1)\right)-V_{1,1}^{(i)}\left(x_{i}(n)\right) \\
& =\lambda_{1}^{(i)}\left(x_{i}(n+1)-x_{i}(n)-x_{i}^{*} \ln \left(\frac{x_{i}(n+1)}{x_{i}(n)}\right)\right) \\
& =\lambda_{1}^{(i)} h\left(x_{i}(n)-x_{i}^{*}\right)\left(r_{i}-b_{i} x_{i}(n)-e_{i} y_{i}\left(n-\tau_{1}(n)\right)\right. \\
& -\frac{p_{i} y_{i}(n)}{1+u_{i} x_{i}(n)+v_{i} y_{i}(n)} \\
& \left.+\sum_{j=1}^{l} a_{i j}\left(\frac{x_{j}(n)}{x_{i}(n)}-\alpha_{i j}\right)\right)+o(h) \\
& =\lambda_{1}^{(i)} h\left(x_{i}(n)-x_{i}^{*}\right)\left(-b_{i}\left(x_{i}(n)-x_{i}^{*}\right)-e_{i}\left(y_{i}\left(n-\tau_{1}(n)\right)-y_{i}^{*}\right)\right. \\
& \left.+\sum_{j=1}^{l} a_{i j}\left(\frac{x_{j}(n)}{x_{i}(n)}-\frac{x_{j}^{*}}{x_{i}^{*}}\right)\right)-\lambda_{1}^{(i)} h\left(x_{i}(n)-x_{i}^{*}\right)\left(\frac{p_{i} y_{i}(n)}{1+u_{i} x_{i}(n)+v_{i} y_{i}(n)}\right. \\
& \left.-\frac{p_{i} y_{i}^{*}}{1+u_{i} x_{i}^{*}+v_{i} y_{i}^{*}}\right)+o(h) \\
& =-\lambda_{1}^{(i)} b_{i} h\left(x_{i}(n)-x_{i}^{*}\right)^{2}-\lambda_{1}^{(i)} e_{i} h\left(x_{i}(n)-x_{i}^{*}\right)\left(y_{i}\left(n-\tau_{1}(n)\right)-y_{i}^{*}\right) \\
& +\sum_{j=1}^{l} a_{i j} \lambda_{1}^{(i)} h x_{j}^{*}\left(\frac{x_{j}(n)}{x_{j}^{*}}-\frac{x_{i}(n)}{x_{i}^{*}}-\frac{x_{j}(n) x_{i}^{*}}{x_{i}(n) x_{j}^{*}}+1\right) \\
& -\frac{\lambda_{1}^{(i)} p_{i} h\left(1+u_{i} x_{i}^{*}\right)\left(x_{i}(n)-x_{i}^{*}\right)\left(y_{i}(n)-y_{i}^{*}\right)}{\left(1+u_{i} x_{i}(n)+v_{i} y_{i}(n)\right)\left(1+u_{i} x_{i}^{*}+v_{i} y_{i}^{*}\right)} \\
& +\frac{\lambda_{1}^{(i)} p_{i} h u_{i} y_{i}^{*}\left(x_{i}(n)-x_{i}^{*}\right)^{2}}{\left(1+u_{i} x_{i}(n)+v_{i} y_{i}(n)\right)\left(1+u_{i} x_{i}^{*}+v_{i} y_{i}^{*}\right)}+o(h) \\
& \leq \lambda_{1}^{(i)} h\left(-b_{i}+\frac{p_{i} u_{i} y_{i}^{*}}{\left(1+u_{i} x_{i}(n)+v_{i} y_{i}(n)\right)\left(1+u_{i} x_{i}^{*}+v_{i} y_{i}^{*}\right)}+\frac{\lambda_{1}^{(i)}}{2}\right)
\end{aligned}
$$




$$
\begin{aligned}
& \times\left(x_{i}(n)-x_{i}^{*}\right)^{2}+\frac{e_{i}^{2} h}{2}\left(y_{i}\left(n-\tau_{1}(n)\right)-y_{i}^{*}\right)^{2} \\
& -\frac{\lambda_{1}^{(i)} p_{i} h\left(1+u_{i} x_{i}^{*}\right)\left(x_{i}(n)-x_{i}^{*}\right)\left(y_{i}(n)-y_{i}^{*}\right)}{\left(1+u_{i} x_{i}(n)+v_{i} y_{i}(n)\right)\left(1+u_{i} x_{i}^{*}+v_{i} y_{i}^{*}\right)} \\
& +\sum_{j=1}^{l} \tilde{a}_{i j} F_{i j}\left(x_{i}(n), x_{j}(n)\right)+o(h)
\end{aligned}
$$

where $F_{i j}\left(x_{i}(n), x_{j}(n)\right)=h\left(\frac{x_{j}(n)}{x_{j}^{*}}-\frac{x_{i}(n)}{x_{i}^{*}}-\frac{x_{j}(n) x_{i}^{*}}{x_{i}(n) x_{j}^{*}}+1\right)$. Similarly, $\Delta V_{2,1}^{(i)}\left(y_{i}(n)\right)$ can be estimated as follows:

$$
\begin{aligned}
& \Delta V_{2,1}^{(i)}\left(y_{i}(n)\right) \\
& =V_{2,1}^{(i)}\left(y_{i}(n+1)\right)-V_{2,1}^{(i)}\left(y_{i}(n)\right) \\
& =\lambda_{2}^{(i)}\left(y_{i}(n+1)-y_{i}(n)-y_{i}^{*} \ln \left(\frac{y_{i}(n+1)}{y_{i}(n)}\right)\right) \\
& =\lambda_{2}^{(i)} h\left(y_{i}(n)-y_{i}^{*}\right)\left(\varepsilon_{i} x_{i}\left(n-\tau_{2}(n)\right)+\frac{q_{i} x_{i}(n)}{1+u_{i} x_{i}(n)+v_{i} y_{i}(n)}\right. \\
& \left.-\gamma_{i}-\delta_{i} y_{i}(n)+\sum_{j=1}^{l} b_{i j}\left(\frac{y_{j}(n)}{y_{i}(n)}-\beta_{i j}\right)\right)+o(h) \\
& =\lambda_{2}^{(i)} h\left(y_{i}(n)-y_{i}^{*}\right)\left(\varepsilon_{i}\left(x_{i}\left(n-\tau_{2}(n)\right)-x_{i}^{*}\right)-\delta_{i}\left(y_{i}(n)-y_{i}^{*}\right)\right. \\
& \left.+\sum_{j=1}^{l} b_{i j}\left(\frac{y_{j}(n)}{y_{i}(n)}-\frac{y_{j}^{*}}{y_{i}^{*}}\right)\right)+\lambda_{2}^{(i)} h\left(y_{i}(n)-y_{i}^{*}\right) \\
& \times\left(\frac{q_{i} x_{i}(n)}{1+u_{i} x_{i}(n)+v_{i} y_{i}(n)}-\frac{q_{i} x_{i}^{*}}{1+u_{i} x_{i}^{*}+v_{i} y_{i}^{*}}\right)+o(h) \\
& =-\lambda_{2}^{(i)} \delta_{i} h\left(y_{i}(n)-y_{i}^{*}\right)^{2}+\lambda_{2}^{(i)} \varepsilon_{i} h\left(y_{i}(n)-y_{i}^{*}\right)\left(x_{i}\left(n-\tau_{2}(n)\right)-x_{i}^{*}\right) \\
& +\sum_{j=1}^{l} b_{i j} \lambda_{2}^{(i)} h y_{j}^{*}\left(\frac{y_{j}(n)}{y_{j}^{*}}-\frac{y_{i}(n)}{y_{i}^{*}}-\frac{y_{j}(n) y_{i}^{*}}{y_{i}(n) y_{j}^{*}}+1\right) \\
& +\frac{\lambda_{2}^{(i)} q_{i} h\left(1+v_{i} y_{i}^{*}\right)\left(x_{i}(n)-x_{i}^{*}\right)\left(y_{i}(n)-y_{i}^{*}\right)}{\left(1+u_{i} x_{i}(n)+v_{i} y_{i}(n)\right)\left(1+u_{i} x_{i}^{*}+v_{i} y_{i}^{*}\right)} \\
& -\frac{\lambda_{2}^{(i)} q h v_{i} x_{i}^{*}\left(y_{i}(n)-y_{i}^{*}\right)^{2}}{\left(1+u_{i} x_{i}(n)+v_{i} y_{i}(n)\right)\left(1+u_{i} x_{i}^{*}+v_{i} y_{i}^{*}\right)}+o(h) \\
& \leq \lambda_{2}^{(i)} h\left(-\delta_{i}+\frac{\lambda_{2}^{(i)}}{2}\right)\left(y_{i}(n)-y_{i}^{*}\right)^{2}+\frac{\varepsilon_{i}^{2} h}{2}\left(x_{i}\left(n-\tau_{2}(n)\right)-x_{i}^{*}\right)^{2} \\
& +\frac{\lambda_{2}^{(i)} q_{i} h\left(1+v_{i} y_{i}^{*}\right)\left(x_{i}(n)-x_{i}^{*}\right)\left(y_{i}(n)-y_{i}^{*}\right)}{\left(1+u_{i} x_{i}(n)+v_{i} y_{i}(n)\right)\left(1+u_{i} x_{i}^{*}+v_{i} y_{i}^{*}\right)} \\
& +\sum_{j=1}^{l} \tilde{b}_{i j} G_{i j}\left(y_{i}(n), y_{j}(n)\right)+o(h)
\end{aligned}
$$


where $G_{i j}\left(y_{i}(n), y_{j}(n)\right)=h \theta\left(\frac{y_{j}(n)}{y_{j}^{*}}-\frac{y_{i}(n)}{y_{i}^{*}}-\frac{y_{j}(n) y_{i}^{*}}{y_{i}(n) y_{j}^{*}}+1\right)$. Then we estimate $\Delta V_{1,2}^{(i)}\left(x_{i}(n)\right)$, $\Delta V_{1,3}^{(i)}\left(x_{i}(n)\right), \Delta V_{2,2}^{(i)}\left(y_{i}(n)\right)$, and $\Delta V_{2,3}^{(i)}\left(y_{i}(n)\right)$ as follows:

$$
\begin{aligned}
& \Delta V_{1,2}^{(i)}\left(x_{i}(n)\right) \\
& =\sigma_{1}^{(i)} \sum_{r=n+1-\tau_{2}(n+1)}^{n}\left(x_{i}(r)-x_{i}^{*}\right)^{2}-\sigma_{1}^{(i)} \sum_{r=n-\tau_{2}(n)}^{n-1}\left(x_{i}(r)-x_{i}^{*}\right)^{2} \\
& =\sigma_{1}^{(i)}\left(\left(x_{i}(n)-x_{i}^{*}\right)^{2}-\left(x_{i}\left(n-\tau_{2}(n)\right)-x_{i}^{*}\right)^{2}\right. \\
& \left.+\sum_{r=n+1-\tau_{2}(n+1)}^{n-1}\left(x_{i}(r)-x_{i}^{*}\right)^{2}-\sum_{r=n+1-\tau_{2}(n)}^{n-1}\left(x_{i}(r)-x_{i}^{*}\right)^{2}\right) \\
& \leq \sigma_{1}^{(i)}\left(\left(x_{i}(n)-x_{i}^{*}\right)^{2}-\left(x_{i}\left(n-\tau_{2}(n)\right)-x_{i}^{*}\right)^{2}\right. \\
& \left.+\sum_{r=n+1-\tau_{M}^{(2)}}^{n-1}\left(x_{i}(r)-x_{i}^{*}\right)^{2}-\sum_{r=n+1-\tau_{m}^{(2)}}^{n-1}\left(x_{i}(r)-x_{i}^{*}\right)^{2}\right) \\
& =\sigma_{1}^{(i)}\left(\left(x_{i}(n)-x_{i}^{*}\right)^{2}-\left(x_{i}\left(n-\tau_{2}(n)\right)-x_{i}^{*}\right)^{2}+\sum_{r=n+1-\tau_{M}^{(2)}}^{n-\tau_{m}^{(2)}}\left(x_{i}(r)-x_{i}^{*}\right)^{2}\right), \\
& \Delta V_{1,3}^{(i)}\left(x_{i}(n)\right) \\
& =\sigma_{1}^{(i)} \sum_{q=n-\tau_{M}^{(2)}+2}^{n-\tau_{m}^{(2)}+1} \sum_{r=q}^{n}\left(x_{i}(r)-x_{i}^{*}\right)^{2}-\sigma_{1}^{(i)} \sum_{q=n-\tau_{M}^{(2)}+1}^{n-\tau_{m}^{(2)}} \sum_{r=q}^{n-1}\left(x_{i}(r)-x_{i}^{*}\right)^{2} \\
& =\sigma_{1}^{(i)}\left(\left(x_{i}(n)-x_{i}^{*}\right)^{2}+\sum_{r=n-\tau_{m}^{(2)}+1}^{n-1}\left(x_{i}^{(k)}(r)-x_{i}^{(k *)}\right)^{2}\right. \\
& \left.-\sum_{r=n-\tau_{M}^{(2)}+1}^{n-1}\left(x_{i}(r)-x_{i}^{*}\right)^{2}+\left(\tau_{M}^{(2)}-\tau_{m}^{(2)}-1\right)\left(x_{i}(n)-x_{i}^{*}\right)^{2}\right) \\
& =\sigma_{1}^{(i)}\left(\left(\tau_{M}^{(2)}-\tau_{m}^{(2)}\right)\left(x_{i}(n)-x_{i}^{*}\right)^{2}-\sum_{r=n+1-\tau_{M}^{(2)}}^{n-\tau_{m}^{(2)}}\left(x_{i}(r)-x_{i}^{*}\right)^{2}\right), \\
& \Delta V_{2,2}^{(i)}\left(y_{i}(n)\right) \\
& \leq \sigma_{2}^{(i)}\left(\left(y_{i}(n)-y_{i}^{*}\right)^{2}-\left(y_{i}\left(n-\tau_{1}(n)\right)-y_{i}^{*}\right)^{2}+\sum_{r=n+1-\tau_{M}^{(1)}}^{n-\tau_{m}^{(1)}}\left(y_{i}(r)-y_{i}^{*}\right)^{2}\right), \\
& \Delta V_{2,3}^{(i)}\left(y_{i}(n)\right) \\
& =\sigma_{2}^{(i)}\left(\left(\tau_{M}^{(1)}-\tau_{m}^{(1)}\right)\left(y_{i}(n)-y_{i}^{*}\right)^{2}-\sum_{r=n+1-\tau_{M}^{(1)}}^{n-\tau_{m}^{(1)}}\left(y_{i}(r)-y_{i}^{*}\right)^{2}\right) .
\end{aligned}
$$


By (7), (8), (9), (10), (11), and (12), it follows that

$$
\begin{aligned}
c_{1}^{(i)} \sum_{r=1}^{3} \Delta V_{1, r}^{(i)}\left(x_{i}(n)\right)+c_{2}^{(i)} \sum_{r=1}^{3} \Delta V_{2, r}^{(i)}\left(y_{i}(n)\right) \\
\leq \lambda_{1}^{(i)} c_{1}^{(i)} h\left(-b_{i}+\frac{p_{i} u_{i} y_{i}^{*}}{\left(1+u_{i} x_{i}^{*}+v_{i} y_{i}^{*}\right)}+\frac{\lambda_{1}^{(i)}}{2}+\frac{\sigma_{1}^{(i)}\left(1+\tau_{M}^{(2)}-\tau_{m}^{(2)}\right)}{\lambda_{1}^{(i)} h}\right) \\
\quad \times\left(x_{i}(n)-x_{i}^{*}\right)^{2}+\lambda_{2}^{(i)} c_{2}^{(i)} h\left(-\delta_{i}+\frac{\lambda_{2}^{(i)}}{2}+\frac{\sigma_{2}^{(i)}\left(1+\tau_{M}^{(1)}-\tau_{m}^{(1)}\right)}{\lambda_{2}^{(i)} h}\right) \\
\quad \times\left(y_{i}(n)-y_{i}^{*}\right)^{2}+\left(\frac{c_{2}^{(i)} \varepsilon_{i}^{2} h}{2}-c_{1}^{(i)} \sigma_{1}^{(i)}\right)\left(x_{i}\left(n-\tau_{2}(n)\right)-x_{i}^{*}\right)^{2} \\
\quad+\left(\frac{c_{1}^{(i)} e_{i}^{2} h}{2}-c_{2}^{(i)} \sigma_{2}^{(i)}\right)\left(y_{i}\left(n-\tau_{1}(n)\right)-y_{i}^{*}\right)^{2} \\
\quad+\sum_{j=1}^{l} c_{1}^{(i)} \tilde{a}_{i j} F_{i j}\left(x_{i}(n), x_{j}(n)\right)+\sum_{j=1}^{l} c_{2}^{(i)} \tilde{b}_{i j} G_{i j}\left(y_{i}(n), y_{j}(n)\right)+o(h) .
\end{aligned}
$$

Because $\left(\mathcal{G}_{1}, \tilde{A}\right)$ and $\left(\mathcal{G}_{2}, \tilde{B}\right)$ are both strongly connected, by Theorem 2.2 in [9], we have $c_{1}^{(i)}>0$ and $c_{2}^{(i)}>0$ for $i \in \mathbb{L}$. Assume that there is $i \in \mathbb{L}$ such that $X_{i}^{(n)} \neq X_{i}^{*}$. For any $\varepsilon$ and $M$ satisfying $0<\varepsilon<M$, we can derive from (13) that, for any $X_{0} \in \mathbb{S}_{M}^{2 l}$ and $X_{0} \neq X^{*}$, there are $\tilde{h}(\varepsilon, M)>0$ and $\mathbb{S}_{M}^{2 l}\left(X^{*}\right) \in \mathbb{R}_{+}^{2 l}$ such that, for any $h \in(0, \tilde{h})$, the following two cases hold.

(i) If $X_{n} \in \mathbb{S}_{M}^{2 l}$ and $\left|X_{i}^{(n)}\right|>\varepsilon / 2$, according to (4) and (5), we have that

$$
\begin{aligned}
c_{1}^{(i)} & \sum_{r=1}^{3} \Delta V_{1, r}^{(i)}\left(x_{i}(n)\right)+c_{2}^{(i)} \sum_{r=1}^{3} \Delta V_{2, r}^{(i)}\left(y_{i}(n)\right) \\
& <\sum_{j=1}^{l} c_{1}^{(i)} \tilde{a}_{i j} F_{i j}\left(x_{i}(n), x_{j}(n)\right)+\sum_{j=1}^{l} c_{2}^{(i)} \tilde{b}_{i j} G_{i j}\left(y_{i}(n), y_{j}(n)\right) .
\end{aligned}
$$

Combining (6), (14) with Theorem 2.2 in [9], we obtain that

$$
\begin{aligned}
\Delta V\left(X_{n}\right)= & \sum_{i=1}^{l}\left(c_{1}^{(i)} \sum_{r=1}^{3} \Delta V_{1, r}^{(i)}\left(x_{i}(n)\right)+c_{2}^{(i)} \sum_{r=1}^{3} \Delta V_{2, r}^{(i)}\left(y_{i}(n)\right)\right) \\
\leq & \sum_{i=1}^{l} \sum_{j=1}^{l} c_{1}^{(i)} \tilde{a}_{i j} F_{i j}\left(x_{i}(n), x_{j}(n)\right)+\sum_{i=1}^{l} \sum_{j=1}^{l} c_{2}^{(i)} \tilde{b}_{i j} G_{i j}\left(y_{i}(n), y_{j}(n)\right) \\
\leq & \sum_{\mathcal{Q}_{1} \in \mathbb{Q}_{1}} W\left(\mathcal{Q}_{1}\right) \sum_{(\rho, r) \in \mathcal{V}\left(\mathcal{C}_{\mathcal{Q}_{1}}\right)} F_{r \rho}\left(x_{r}(n), x_{\rho}(n)\right) \\
& +\sum_{\mathcal{Q}_{2} \in \mathbb{Q}_{2}} W\left(\mathcal{Q}_{2}\right) \sum_{(\rho, r) \in \mathcal{V}\left(\mathcal{C}_{\mathcal{Q}_{2}}\right)} G_{r \rho}\left(y_{r}(n), y_{\rho}(n)\right),
\end{aligned}
$$

where $n \in \mathbb{N}, \mathbb{Q}_{1}$ and $\mathbb{Q}_{2}$ are the sets of all spanning unicyclic graphs of $\left(\mathcal{G}_{1}, A\right)$ and $\left(\mathcal{G}_{2}, B\right)$, $W\left(\mathcal{Q}_{1}\right)$ and $W\left(\mathcal{Q}_{2}\right)$ stand for the weight of $\mathcal{Q}_{1}$ and $\mathcal{Q}_{2}, \mathcal{C}_{\mathcal{Q}_{1}}$ and $\mathcal{C}_{\mathcal{Q}_{2}}$ represent the directed cycle of $\mathcal{Q}_{1}$ and $\mathcal{Q}_{2}$, respectively. 
Furthermore, for each directed cycle $\mathcal{C}$ of $\left(\mathcal{G}_{1}, \tilde{A}\right)$ and $\left(\mathcal{G}_{2}, \tilde{B}\right)$, for all $x_{i}, x_{j}, y_{i}, y_{j} \in \mathbb{R}_{+}^{1}$, it holds that

$$
\begin{aligned}
\sum_{(j, i) \in \mathcal{V}(\mathcal{C})} F_{i j}\left(x_{i}(n), x_{j}(n)\right) & =h \sum_{(j, i) \in \mathcal{V}(\mathcal{C})}\left(\frac{x_{j}(n)}{x_{j}^{*}}-\frac{x_{i}(n)}{x_{i}^{*}}-\frac{x_{j}(n) x_{i}^{*}}{x_{i}(n) x_{j}^{*}}+1\right) \\
& \leq h \sum_{(j, i) \in \mathcal{V}(\mathcal{C})}\left(-\frac{x_{i}(n)}{x_{i}^{*}}+\ln \frac{x_{i}(n)}{x_{i}^{(*)}}+\frac{x_{j}(n)}{x_{j}^{(*)}}-\ln \frac{x_{j}(n)}{x_{j}^{(*)}}\right) \\
& =0 .
\end{aligned}
$$

By the same way, we can get $\sum_{(j, i) \in \mathcal{V}(\mathcal{C})} G_{i j}\left(y_{i}(n), y_{j}(n)\right) \leq 0$.

Because $W\left(\mathcal{Q}_{1}\right)>0$ and $W\left(\mathcal{Q}_{2}\right)>0$, it is easy to see that $\Delta V\left(X_{n}\right)<0$. That is to say $\left|X_{n+1}\right|<\left|X_{n}\right|$.

(ii) If $X_{n} \in \mathbb{S}_{M}^{2 l}$ and for all $k \in \mathbb{L},\left|X_{k}^{(n)}\right|<\varepsilon / 2$, by (3), we have $\left|X_{n+1}\right|<\varepsilon$ holds.

Thus, the results of Theorem 1 could be obtained.

Remark 1 Compared with previous references, some differences and novelties of our results should be mentioned here. In [3, 4], the stability of predator-prey systems with Beddington-DeAngelis functional response was studied, but the impact of dispersal among prey and predators was not considered. In [8, 9], predator-prey systems were investigated where only prey dispersal was considered. Because both prey and predators can disperse among multiple groups, in $[10,11]$, multiple disperse was taken into consideration by employing multi-digraph-based approach. However, time-varying delay should not be ignored since a predator can capture prey if and only if it reaches capturing age. In this paper, we study the stability of a predator-prey system with Beddington-DeAngelis functional response which contains both time-varying delay and multiple dispersal, which is more close to the realistic situation.

Remark 2 Recently, the graph-theoretic technique has been used to analyze a single patch population model and a coupled oscillators model; see [15, 16] and [17] for example. In [18-26], the stability of coupled systems on networks was analyzed effectively by the graph-theoretic technique. These works all considered a single coupling situation where the dispersal or coupling of only one component was studied. Hence the model in the above literature was built on a single digraph. Considering the practical meaning of multiple dispersal, the authors studied multi-patch model with multiple dispersal by multidigraph theory $[10,11]$. And in [27], Guo et al. extended this method to study the inputto-state stability for stochastic multi-group models with multi-dispersal. However, owning to time-varying delay, the analysis method proposed in $[10,11]$ is ineffective since the time-varying delay cannot be dealt with. To overcome this obstacle, we consider the Lyapunov-Krasovskii method, i.e., four more functions $V_{1,2}^{(i)}, V_{1,3}^{(i)}, V_{2,2}^{(i)}$, and $V_{2,3}^{(i)}$ are constructed to deal with time-varying delay.

Remark 3 In Theorem 1, two points should be stressed for the dispersal topologies of predators and prey: (I) The dispersal topologies should be strongly connected, which is helpful to cope with the dispersal among patches; (II) The dispersal topologies of predators and prey are proportional, i.e., $c_{1}^{(i)}=\theta c_{2}^{(i)}$, which is helpful to deal with the cross term between predators and prey. In fact, for point (I), it could be well solved by layering the 
large-scale dispersal networks into several strongly connected parts; for more details, one can refer to [18]. For point (II), it is still difficult for multiple dispersal model if the dispersal topologies are not proportional.

Remark 4 In this paper, we require that the dispersal networks of prey and predators among patches are strongly connected. Biologically, this means that prey or a predator from patch $i$ can always arrive at patch $j$. If the dispersal networks are not strongly connected, there exists at least one patch (set as patch $i^{*}$ ) such that prey or predators from other patches cannot reach patch $i^{*}$, but the prey or predators in patch $i^{*}$ can always leave patch $i^{*}$ to other patches. Hence, the prey or predators in patch $i^{*}$ may die out. Therefore, it is meaningful to require the strong connectedness of dispersal networks from biological viewpoint.

\section{Numerical example}

In this section, we consider a predator-prey model with dispersal among four patches and the parameters are selected as follows:

$$
\begin{aligned}
& r_{i}=4.2, \quad b_{i}=2.0, \quad e_{i}=0.1, \quad \gamma_{i}=0.05, \quad \delta_{i}=0.95, \quad \varepsilon_{i}=0.475, \\
& u_{i}=1, \quad v_{i}=1, \quad p_{i}=0.4, \quad q_{i}=0.1, \quad i=1,2,3,4 .
\end{aligned}
$$

The dispersal coefficients for prey and predators are chosen as $a_{12}=0.2549, a_{13}=0.1755$, $a_{21}=0.2879, a_{23}=0.2085, a_{31}=0.2085, a_{34}=0.2879, a_{43}=0.3749, b_{12}=0.05353, b_{13}=$ $0.03686, b_{21}=0.06046, b_{23}=0.0438, b_{31}=0.0438, b_{34}=0.06046, b_{43}=0.07873$. Except these, other dispersal coefficients $a_{i j}=b_{i j}=0$. See the dispersal networks in Fig. 1.

Let $h=0.001, \sigma_{1}=\sigma_{1}=0.001, \tau_{1}(n)=\tau_{2}(n)=[1.5+0.5 \sin (n)]$. To begin with, when $\alpha_{i j}=$ $1, \beta_{i j}=1, i, j=1,2,3,4$, we have

$$
\left\{\begin{array}{l}
r_{i}-b_{i} x_{i}^{*}-e_{i} y_{i}^{*}-\frac{p_{i} y_{i}^{*}}{1+u_{i} x_{i}^{*}+v_{i} y_{i}}=0, \\
-\gamma_{i}-\delta_{i} y_{i}^{*}+\varepsilon_{i} x_{i}^{*}+\frac{q_{i} x_{i}^{*}}{1+u_{i} x_{i}^{*}+v_{i} y_{i}^{*}}=0,
\end{array} \quad i=1,2,3,4\right.
$$

By simple calculation, we obtain that $x_{i}^{*}=2, y_{i}^{*}=1, i=1,2,3,4$, which implies that the fixed point is $X^{*}=(2,1,2,1, \ldots, 2,1)_{8 \times 1}^{\mathrm{T}}$. By definitions $\tilde{a}_{i j}=a_{i j} q_{i}\left(1+v_{i} y^{*}\right) x_{j}^{*}$ and $\tilde{b}_{i j}=b_{i j} p_{i}\left(1+u_{i} x^{*}\right) y_{j}^{*}$, we have that $\tilde{a}_{12}=0.1019, \tilde{a}_{13}=0.0702, \tilde{a}_{21}=0.115, \tilde{a}_{23}=0.0834$, $\tilde{a}_{31}=0.0834, \tilde{a}_{34}=0.1151, \tilde{a}_{43}=0.1499, \tilde{b}_{12}=0.06424, \tilde{b}_{13}=0.0442, \tilde{b}_{21}=0.0725, \tilde{b}_{23}=$ $0.0525, \tilde{b}_{31}=0.05256, \tilde{b}_{34}=0.0725, \tilde{b}_{43}=0.0944$. By calculation, we have $\theta=c_{1}^{(i)} / c_{2}^{(i)}=4$, $i=1,2,3,4$.

From the above dispersal coefficients, it is clear that digraphs $\left(\mathcal{G}_{1}, A\right)$ and $\left(\mathcal{G}_{2}, B\right)$ are strongly connected. All the conditions of Theorem 1 have been verified. Hence, we can conclude that the fixed point $X^{*}$ remains stable in the positive cone $\mathbb{R}_{+}^{8}$. The initial values are given as $\left(x_{1}(n), y_{1}(n), \ldots, x_{4}(n), y_{4}(n)\right)^{\mathrm{T}}=(0.8+0.1 n, 0.53+0.2 n, 1.7+0.2 n, 0.71+$ $0.1 n, 2.1+0.1 n, 1.24+0.2 n, 2.4+0.1 n, 1.5+0.1 n)^{\mathrm{T}}$, where $n=-2,-1,0$. Then the corresponding simulation results are shown in Fig. 3. Figure 3 illustrates that the fixed point $X^{*}$ of system (3) is stable, which shows the effectiveness of our theoretical results.

\section{Conclusion}

This paper studied the stability of a discrete-time multi-patch Beddington-DeAngelis type predator-prey model with time-varying delay, where the dispersal of both predators 


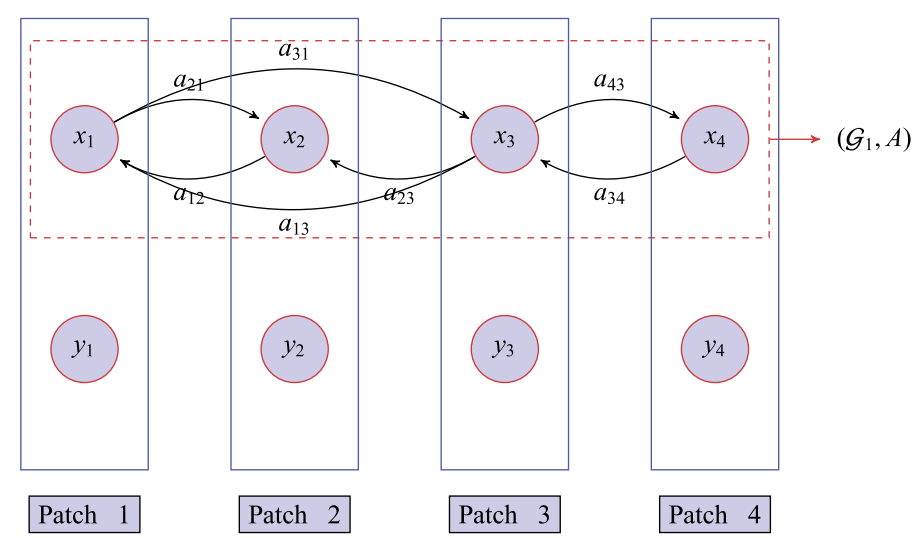

Figure 3 The solution of system (3) on four patches with time step size $h=0.001$ and the dispersal networks as in Figure 1

and prey was considered. By employing the Lyapunov-Krasovskii method and the graphtheoretical technique, a stability criterion was derived. Finally, an example with numerical simulation was given to demonstrate the effectiveness of the obtained results. Because noise disturbance in our real life is ubiquitous [28-30], in the future, we will try to take the effect of noise disturbance into our model.

\section{Acknowledgements}

The authors would like to thank Dr. Sen Li for useful discussion about the numerical simulations.

Funding

This work was supported by the National Natural Science Foundation of China (No. 61401283) and Educational Commission of Guangdong Province, China (No. 2014KTSCX113).

Availability of data and materials

Not applicable.

\section{Competing interests}

The authors declare that they have no competing interests.

Authors' contributions

All authors contributed equally to this manuscript. All authors read and approved the final manuscript.

\section{Author details}

'Shenzhen Key Laboratory of Advanced Machine Learning and Application, College of Mathematics and Statistics, Shenzhen University, Shenzhen, China. ${ }^{2}$ Department of Mathematics, Harbin Institute of Technology (Weihai), Weihai, China.

\section{Publisher's Note}

Springer Nature remains neutral with regard to jurisdictional claims in published maps and institutional affiliations.

Received: 1 September 2018 Accepted: 4 October 2019 Published online: 21 October 2019

\section{References}

1. Liu, M., Bai, C.: Global asymptotic stability of a stochastic delayed predator-prey model with Beddington-DeAngelis functional response. Appl. Math. Comput. 226, 581-588 (2014)

2. Qiu, H., Liu, M., Wang, K.: Dynamics of a stochastic predator-prey system with Beddington-DeAngelis functional response. Appl. Math. Comput. 219, 2303-2312 (2017)

3. Liu, M., Wang, K.: Global stability of stage-structured predator-prey models with Beddington-DeAngelis functional response. Commun. Nonlinear Sci. Numer. Simul. 16, 3792-3797 (2011)

4. Liu, M., Wang, K.: Global stability of a nonlinear stochastic predator-prey system with Beddington-DeAngelis functional response. Commun. Nonlinear Sci. Numer. Simul. 16, 1114-1121 (2011)

5. Fan, M., Kuang, Y.: Dynamics of a nonautonomous predator-prey system with the Beddington-DeAngelis functional response. J. Math. Anal. Appl. 295, 15-39 (2014) 
6. Beddington, J.R.: Mutual interference between parasites or predators and its effect on searching efficiency. J. Anim. Ecol. 44, 331-341 (1975)

7. DeAngelis, D.L., Goldsten, R.A., Neill, R.: A model for trophic interaction. Ecology 56, 881-892 (1975)

8. Su, H., Li, W., Wang, K.: Global stability analysis of discrete-time coupled systems on networks and its applications. Chaos 22, $033135(2012)$

9. Li, M.Y., Shuai, Z.: Global-stability problem for coupled systems of differential equation on networks. J. Differ. Equ. 248, $1-20(2010)$

10. Su, H., Wang, P., Ding, X.: Stability analysis for discrete-time coupled systems with multi-diffusion by graph-theoretic approach and its application. Discrete Contin. Dyn. Syst., Ser. B 21, 253-269 (2016)

11. Zhang, C., Li, W., Wang, K.: Graph-theoretic approach to stability of multi-group models with dispersal. Discrete Contin. Dyn. Syst., Ser. B 20, 259-280 (2015)

12. Lu, Z., Takeuchi, Y.: Global asymptotic behavior in single-species discrete diffusion systems. J. Math. Biol. 32, 67-77 (1993)

13. West, D.B.: Introduction to Graph Theory. Prentice Hall, Upper Saddle River (1996)

14. Wang, W., Zhao, X.: An epidemic model in a patchy environment. Math. Biosci. 190, 97-112 (2004)

15. Geng, J., Liu, M., Zhang, Y.: Stability of a stochastic one-predator-two-prey population model with time delays. Commun. Nonlinear Sci. Numer. Simul. 53, 65-82 (2017)

16. Jin, Y., Liu, M.: Population dynamical behavior of a two-predator one-prey stochastic model with time delay. Discrete Contin. Dyn. Syst. 37, 2513-2538 (2017)

17. Qiao, Y., Huang, Y., Chen, M.: A graph-theoretic approach to global input-to-state stability for coupled control systems. Adv. Differ. Equ. 2017, 129 (2017)

18. Liu, S., Li, W.: Outer synchronization of delayed coupled systems on networks without strong connectedness: a hierarchical method. Discrete Contin. Dyn. Syst., Ser. B 23, 837-859 (2018)

19. Li, S., Su, H., Ding, X.: Synchronized stationary distribution of hybrid stochastic coupled systems with applications to coupled oscillators and a Chua's circuits network. J. Franklin Inst. Eng. Appl. Math. 355, 8743-8765 (2018)

20. Li, S., Zhang, B., Li, W.: Stabilisation of multiweights stochastic complex networks with time-varying delay driven by G-Brownian motion via aperiodically intermittent adaptive control. Int. J. Control (2019). https://doi.org/10.1007/s001090000086

21. Wang, P., Feng, J., Su, H.: Stabilization of stochastic delayed networks with Markovian switching and hybrid nonlinear coupling via aperiodically intermittent control. Nonlinear Anal. Hybrid Syst. 32, 115-130 (2019)

22. Wang, P., Jin, W., Su, H.: Synchronization of coupled stochastic complex-valued dynamical networks with time-varying delays via aperiodically intermittent adaptive control. Chaos 28, 043114 (2018)

23. Wu, Y., Wang, C., Li, W.: Generalized quantized intermittent control with adaptive strategy on finite-time synchronization of delayed coupled systems and applications. Nonlinear Dyn. 95(2), 1361-1377 (2019)

24. Liu, Y., Li, W., Feng, J.: The stability of stochastic coupled systems with time-varying coupling and general topology structure. IEEE Trans. Neural Netw. Learn. Syst. 29(9), 4189-4200 (2018)

25. Xu, Y., Zhou, H., Li, W.: Stabilisation of stochastic delayed systems with Lévy noise on networks via periodically intermittent control. Int. J. Control (2018). https://doi.org/10.1080/00207179.2018.1479538

26. Zhou, H., Zhang, Y., Li, W.: Synchronization of stochastic Levy noise systems on a multi-weights network and its applications of Chua's circuits. IEEE Trans. Circuits Syst. I, Regul. Pap. 66(7), 2709-2722 (2019)

27. Sowmiya, C., Raja, R., Cao, J., Rajchakit, G., Alsaedi, A.: Enhanced robust finite-time passivity for Markovian jumping discrete-time BAM neural networks with leakage delay. Adv. Differ. Equ. 2017, 318 (2017)

28. Zhu, Q:: Stabilization of stochastic nonlinear delay systems with exogenous disturbances and the event-triggered feedback control. IEEE Trans. Autom. Control 64(9), 3764-3771 (2019)

29. Zhu, Q.: Stability analysis of stochastic delay differential equations with Lévy noise. Syst. Control Lett. 118, 62-68 (2018)

30. Zhang, M., Zhu, Q:: New criteria of input-to-state stability for nonlinear switched stochastic delayed systems with asynchronous switching. Syst. Control Lett. 129, 43-50 (2019)

\section{Submit your manuscript to a SpringerOpen ${ }^{\circ}$ journal and benefit from:}

- Convenient online submission

- Rigorous peer review

- Open access: articles freely available online

- High visibility within the field

- Retaining the copyright to your article

Submit your next manuscript at $\gg$ springeropen.com 